\title{
Control Schemes for Passive Teleoperation Systems over Wide Area Communication Networks with Time Varying Delay
}

\author{
Shafiqul Islam ${ }^{1,2,8}$ Xiaoping P. Liu ${ }^{3}$ Abdulmotaleb El Saddik ${ }^{1,4}$ Lakmal Seneviratne $^{5,6}$ Jorge Dias $^{7,8}$ \\ ${ }^{1}$ School of Information Technology and Engineering, University of Ottawa, Ottawa K1N 6N5, Canada \\ ${ }^{2}$ School of Engineering and Applied Sciences, Harvard University, Cambridge, MA 02138, USA \\ ${ }^{3}$ Department of Systems and Computer Engineering, Carleton University, Ottawa K1S5B6, Canada \\ ${ }^{4}$ Electrical Engineering, New York University, AD 129188, UAE \\ ${ }^{5}$ Division of Engineering, Kings College London, UK \\ ${ }^{6}$ Robotics and Mechanical Engineering, Khalifa University of Science \& Technology, AD 127788, UAE \\ ${ }^{7}$ Institute of Systems and Robotics, University of Coimbra, Portugal \\ ${ }^{8}$ Robotics and Electrical and Computer Engineering, Khalifa University of Science \& Technology, AD 127788, UAE
}

\begin{abstract}
In this paper, the problem of time varying telecommunication delays in passive teleoperation systems is addressed. The design comprises delayed position, velocity and position-velocity signals with the local position and velocity signals of the master and slave manipulators. Nonlinear adaptive control terms are employed locally to cope with uncertain parameters associated with the gravity loading vector of the master and slave manipulators. Lyapunov-Krasovskii function is employed for three methods to establish asymptotic tracking property of the closed loop teleoperation systems. The stability analysis is derived for both symmetrical and unsymmetrical time varying delays in the forward and backward communication channel that connects the local and remote sites. Finally, evaluation results are presented to illustrate the effectiveness of the proposed design for real-time applications.
\end{abstract}

Keywords: Teleoperation, telerobotics, Lyapunov-Krasovskii functional, time varying delay, passive systems.

\section{Introduction}

Over the past decades bilateral teleoperation control technology has been attracted by many researchers around the world, see for example, [1-25, and many others]. The main goal in most reported control mechanism for teleoperation systems is to ensure stability and transparency of the whole closed loop system in the presence of time delay. These results can be broadly classified into two categories as passive and nonpassive teleoperation systems. The control system for nonpassive teleoperation can be found in [1-4]. Polushin et al. ${ }^{[1,2]}$ developed teleoperation systems in the presence of nonpassive human and environment input forces under symmetrical time varying delay. Small gain theorem was employed to show the position and velocity synchronization errors between master and slave manipulators provided that the human and environment input forces are bounded. Most recently, authors in [3,4] have introduced control methods for nonpassive bilateral teleoperation systems in the presence of unsymmetrical time varying delay. The stability analysis of these methods was based on using strict linear matrix inequality. Control mechanisms for passive teleoperation systems have been extensively studied by many researchers, see for example, [5-25]. Historical survey and comparison with different teleoperation control algorithms can be traced from $[5,6]$. Classical teleoperation schemes using passivity theorem and scattering approach have been proposed in $[7-11]$. Li and Song ${ }^{[12]}$ developed virtual environment modeling and correction technique for force reflecting teleoperation with constant time delays.

Manuscript received January 21, 2013; revised May 2, 2013

This work was supported by Natural Sciences and Engineering Research Council of Canada (NSERC) Research Fellowship, Canada Research Chairs Program and University of Ottawa Research Chair Program.
Authors in [13] developed a control scheme for Internet based teleoperation systems using wave variable approach under time varying delays. Bate et al. ${ }^{[14]}$ proposed control mechanism to reduce the reflection from wave based passive teleoperation systems. The stability analysis for wave variable method introduced in $[12-14]$ was established for the single degree of freedom (DOF) linearized master and slave manipulators. Alise et al. ${ }^{[15]}$ extended such wave variable mechanism to multiple-DOF systems. In [16, 17], authors presented teleoperation systems in the presence of time varying communication delays. Lee and Spong ${ }^{[18]}$ have presented proportional-derivative control scheme for passive teleoperation systems for position coordination of the master and slave manipulator. Authors in [19] have showed that the stability condition established in [18] can be obtained by using only delayed position signals based teleoperation system. However, the control design parameters require to satisfy specific conditions to ensure closed loop stability of the closed loop teleoperation system. Lee and Huang ${ }^{[20]}$ developed passive set-position modulation framework for Internet based teleoperation systems with time varying delay. In [21], authors presented adaptive tracking system for uncertain nonlinear systems with time varying delays and unmodeled dynamics by using radial basis functional networks.

We can notice from the structure of the delays that most reported passive teleoperators are based on the assumption that the time delays are either constant or time varying nature. Moreover, the time delays in passive designs are assumed to be of symmetrical nature which may not be realistic in bilateral teleoperation applications. In practice, however, the data packet delays, i.e., the time required for the data packets to reach the remotely located sites and data packet returns to the local sites may be of unsymmet- 
rical nature which varies with respect to the network load, number of the traversed nodes and distance ${ }^{[3,4,22]}$.

In this paper, we address the stability problem of passive teleoperation systems under symmetrical and unsymmetrical time varying delays. The teleoperators are designed by coupling local and remote sites via delaying position and position-velocity signals of the master and slave manipulators. The input algorithms also combine local position and velocity signals with nonlinear adaptive terms to cope with the parametric uncertainty associated with the gravity loading vector. In our first design, we introduce passive teleoperation systems under symmetrical time varying delays. In second design, we develop teleoperators depending on unsymmetrical nature of time varying delays. LyapunovKrasovskii function is used to analyze the asymptotic tracking property of the closed loop teleoperation systems. These stability properties are illustrated by conducting simulation studies on a 2-DOF master-slave teleoperation systems.

The rest of the paper is organized as follows: Section 2 describes the dynamics of the teleoperation system. Sections 3 and 4 introduce teleoperation strategies under symmetrical and unsymmetrical time varying communication delays. A detailed convergence analysis is also given in Sections 3 and 4. Simulation example is presented in Section 5. Finally, Section 6 concludes the paper.

\section{Model dynamics}

\subsection{Master and slave manipulator dynam- ics}

Let us first consider the equation of motion for $n$-links master and slave manipulators with human and environment input forces

$$
\begin{aligned}
& M_{m}\left(q_{m}\right) \ddot{q}_{m}+C_{m}\left(q_{m}, \dot{q}_{m}\right) \dot{q}_{m}+G_{m}\left(q_{m}\right)=\tau_{m}+F_{h} \\
& M_{s}\left(q_{s}\right) \ddot{q}_{s}+C_{s}\left(q_{s}, \dot{q}_{s}\right) \dot{q}_{s}+G_{s}\left(q_{s}\right)=\tau_{s}-F_{e}
\end{aligned}
$$

where $m$ and $s$ represent the master and slave systems respectively, $\ddot{q}_{m} \in \mathbf{R}^{n}, \dot{q}_{m} \in \mathbf{R}^{n}, q_{m} \in \mathbf{R}^{n}$ and $\ddot{q}_{s} \in \mathbf{R}^{n}$, $\dot{q}_{s} \in \mathbf{R}^{n}, q_{s} \in \mathbf{R}^{n}$ are the joint acceleration, velocity and position, $M_{m}\left(q_{m}\right) \in \mathbf{R}^{n \times n}$ and $M_{s}\left(q_{s}\right) \in \mathbf{R}^{n \times n}$ are the symmetric and uniformly positive definite inertia matrices, $C_{m}\left(q_{m}, \dot{q}_{m}\right) \dot{q}_{m} \in \mathbf{R}^{n}$ and $C_{s}\left(q_{s}, \dot{q}_{s}\right) \dot{q}_{s} \in \mathbf{R}^{n}$ are the coriolis and centrifugal loading vectors, $G_{m}\left(q_{m}\right) \in \mathbf{R}^{n}$ and $G_{s}\left(q_{s}\right) \in \mathbf{R}^{n}$ is the gravitational loading vector, $\tau_{m} \in \mathbf{R}^{n}$ and $\tau_{s} \in \mathbf{R}^{n}$ are applied control input vector, and $F_{h} \in \mathbf{R}^{n}$ and $F_{e} \in \mathbf{R}^{n}$ denote the operational torque applied to the master by human operator and environmental torque applied to slave manipulators, respectively. The teleoperation systems (1), (2) can be written as

$$
\begin{aligned}
& \ddot{q}_{m}=M_{m}^{-1}\left(q_{m}\right)\left[\tau_{m}+F_{h}-C_{m}\left(q_{m}, \dot{q}_{m}\right) \dot{q}_{m}-G_{m}\left(q_{m}\right)\right] \\
& \ddot{q}_{s}=M_{s}^{-1}\left(q_{s}\right)\left[\tau_{s}-F_{e}-C_{s}\left(q_{s}, \dot{q}_{s}\right) \dot{q}_{s}-G_{s}\left(q_{s}\right)\right] .
\end{aligned}
$$

Throughout our stability analysis, we will use the following dynamical property of master and slave manipulators ${ }^{[23]}$

Property 1. The matrices $\dot{M}_{m}\left(q_{m}\right)-2 C_{m}\left(q_{m}, \dot{q}_{m}\right)$ and $\dot{M}_{s}\left(q_{s}\right)-2 C_{s}\left(q_{s}, \dot{q}_{s}\right)$ are skew symmetric.

Property 2. The mass matrices $M_{m}\left(q_{m}\right)$ and $M_{s}\left(q_{s}\right)$ are the symmetric, bounded and positive definite satisfying the inequality $\left\|M_{m}\left(q_{m}\right)\right\| \leqslant \beta_{m}$ and $\left\|M_{s}\left(q_{s}\right)\right\| \leqslant \beta_{s}$ with $\beta_{m}>0$ and $\beta_{s}>0$.

Property 3. If the signals $\ddot{q}_{m}$ and $\ddot{q}_{s}$ are bounded, then $\dot{C}_{m}\left(q_{m}, \dot{q}_{m}\right)$ and $\dot{C}_{s}\left(q_{s}, \dot{q}_{s}\right)$ are bounded.

\subsection{Interaction forces between human- master and between slave-environment}

The interaction forces between human and master and between slave and environment play a significant role to achieve desired transparency and stability of the closed loop teleoperation systems. These forces can be modeled as a second-order mass-spring-damper system ${ }^{[24]}$. It is also possible to use simpler models to estimate interaction forces, such as, pure stiffness or dampening-stiffness ${ }^{[25]}$. In this work, the interaction forces are estimated by using springdamper systems as $[4,18-20,22,25]$

$$
\begin{aligned}
& F_{h}=-S_{m} q_{m}-D_{m} \dot{q}_{m} \\
& F_{e}=S_{s} q_{s}+D_{s} \dot{q}_{s}
\end{aligned}
$$

where $S_{m} \in \mathbf{R}^{n \times n}$ and $D_{m} \in \mathbf{R}^{n \times n}$ represent stiffness and damping constant matrices for the human operators and $S_{s} \in \mathbf{R}^{n \times n}$ and $D_{s} \in \mathbf{R}^{n \times n}$ defines stiffness and damping constants matrices for the remote environment.

\section{Algorithm design and stability anal- ysis}

In this work, we design two control strategies for passive teleoperation systems and analyze their stability properties in the presence of symmetrical and unsymmetrical time varying telecommunication delays. In our analysis, the data transmission delays in the forward communication channel from master to slave platforms and backward communication channel from slave to master platforms are defined as $\gamma_{d m}(t)$ and $\gamma_{d s}(t)$, respectively.

We first consider that the time delays in forward and backward communication channels are symmetrical as $\gamma_{d m}(t)=\gamma_{d s}(t)=\gamma_{d}(t)$. The input for the systems $(3)$ is then designed by comprising delayed position signal with the local position, velocity and adaptive control term for gravity compensation as

$$
\begin{aligned}
& \tau_{m}=-K_{P m}\left(q_{m}-q_{s}\left(t-\gamma_{d}(t)\right)\right)-K_{D m} \dot{q}_{m}+\hat{G}_{m}\left(q_{m}\right) \\
& \dot{\tilde{\theta}}_{m}=-\Gamma_{m} Y_{m}\left(q_{m}\right) \dot{q}_{m} \\
& \tau_{s}=K_{P s}\left(q_{m}\left(t-\gamma_{d}(t)\right)-q_{s}\right)-K_{D s} \dot{q}_{s}+\hat{G}_{s}\left(q_{s}\right) \\
& \dot{\tilde{\theta}}_{s}=-\Gamma_{s} Y_{s}\left(q_{s}\right) \dot{q}_{s}
\end{aligned}
$$

where $K_{P m}>0, K_{D m}>0, K_{P s}>0, K_{D s}>$ $0, \quad Y_{m}\left(q_{m}\right) \tilde{\theta}_{m}=\left(\hat{G}_{m}\left(q_{m}\right)-G_{m}\left(q_{m}\right)\right), \quad Y_{s}\left(q_{s}\right) \tilde{\theta}_{s}=$ $\left(\hat{G}_{s}\left(q_{s}\right)-G_{s}\left(q_{s}\right)\right), \tilde{\theta}_{m}=\left(\hat{\theta}_{m}-\theta_{m}\right), \tilde{\theta}_{s}=\left(\hat{\theta}_{s}-\theta_{s}\right)$, $\Gamma_{m} \in \mathbf{R}^{m \times m}$ and $\Gamma_{s} \in \mathbf{R}^{s \times s}$ are the positive definite constant matrices. The control scheme (6) is depicted in Fig. 1 with $\gamma_{d m}(t)=\gamma_{d s}(t)=\gamma_{d}(t)$. Parameter adaptation mechanism given above may cause discontinuous property in the parameter estimates even after parameter converges to the actual one. To ensure that the parameters $\theta_{m}$ and $\theta_{s}$ remain bounded over the given compact sets $\theta_{m} \in \Omega_{m}$ and $\theta_{s} \in \Omega_{s}$, a projection algorithm can be used to limit the parameter estimates. For this, we define $\hat{\Omega}_{m}$ and $\hat{\Omega}_{s}$ such that $\hat{\Omega}_{m} \subset \Omega_{m}$ and $\hat{\Omega}_{s} \subset \Omega_{s}$ with $\Omega_{m}=\left\{x_{m i} \leqslant \theta_{m i} \leqslant y_{m i}\right\}$ and $\Omega_{s}=\left\{x_{s i} \leqslant \theta_{s i} \leqslant y_{s i}\right\}$. We also define $\Omega_{\delta m}$ and $\Omega_{\delta s}$ as $\Omega_{\delta m}=\left\{x_{m i}-\delta_{m} \leqslant \theta_{m i} \leqslant y_{m i}-\delta_{m}\right\}$ and $\Omega_{\delta s}=\left\{x_{s i}-\delta_{s} \leqslant \theta_{s i} \leqslant y_{s i}-\delta_{s}\right\}$ where $\delta_{m}$ and $\delta_{s}$ are chosen such that $\hat{\Omega}_{m} \subset \Omega_{\delta m}$ and $\hat{\Omega}_{s} \subset \Omega_{\delta s}$. Then, the projection of the parameter adaptation rule can be written as $\dot{\hat{\theta}}_{m}=\operatorname{Proj}\left(\hat{\theta}_{m}, \Phi_{m}\right)$ and $\dot{\hat{\theta}}_{s}=\operatorname{Proj}\left(\hat{\theta}_{s}, \Phi_{s}\right)$ with 


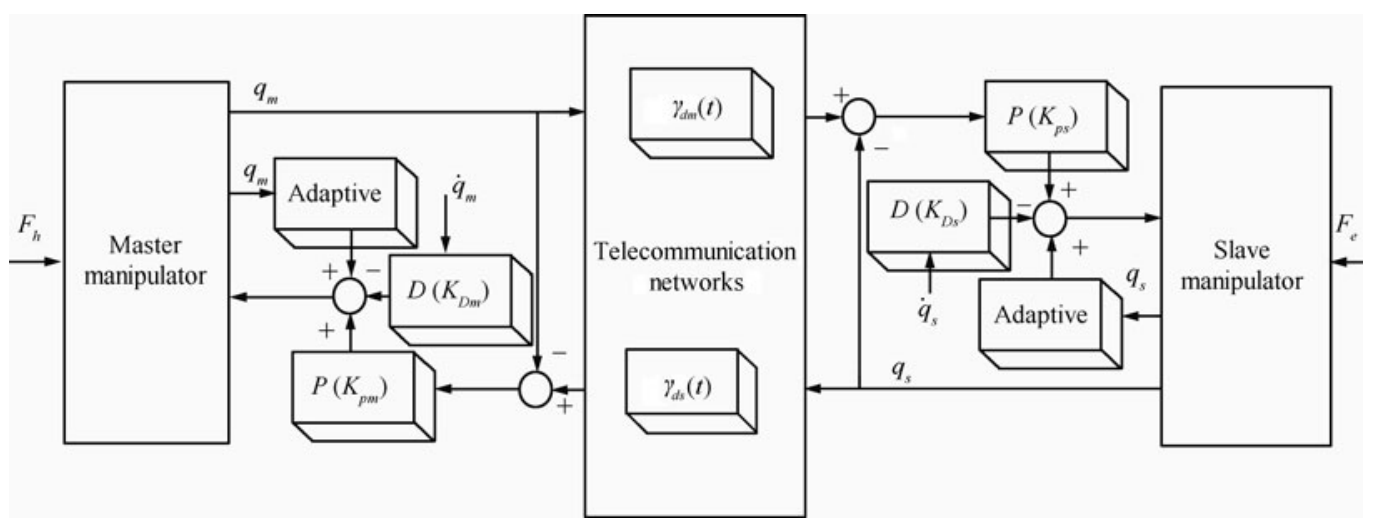

Fig. 1 Control scheme for bilateral teleoperation systems with delayed position signals for symmetrical case $\gamma_{d m}(t)=\gamma_{d s}(t)=\gamma_{d}(t)$ and unsymmetrical case $\gamma_{d m}(t) \neq \gamma_{d s}(t)$

$\left[\operatorname{Proj}\left(\hat{\theta}_{k}, \Phi_{k}\right)\right]_{i}= \begin{cases}\Gamma_{k} \Phi_{k i}, & \text { if } x_{k i} \leqslant \hat{\theta}_{k i} \leqslant y_{k i} \text { or } \\ & \text { if } \hat{\theta}_{k i}>y_{k i} \text { and } \Phi_{k i} \leqslant 0 \text { or } \\ & \text { if } \hat{\theta}_{k i}<x_{k i} \text { and } \Phi_{k i} \geqslant 0 \\ \Gamma_{k} \bar{\Phi}_{k i}, & \text { if } \hat{\theta}_{k i}>y_{k i} \text { and } \Phi_{k i}>0 \\ \Gamma_{k} \breve{\Phi}_{k i}, & \text { if } \hat{\theta}_{k i}<x_{k i} \text { and } \Phi_{k i}<0\end{cases}$

where $\bar{\Phi}_{k i}=\left[1+\frac{y_{k i}-\theta_{k i}}{\delta_{k}}\right] \Phi_{k i}, \breve{\Phi}_{k i}=\left[1+\frac{\theta_{k i}-x_{k i}}{\delta_{k}}\right] \Phi_{k i}$ and $\Phi_{k i}$ is the $i$-th element of the column matrix $-Y_{k}^{\mathrm{T}}\left(q_{k}\right) \dot{q}_{k}$ with $k=m, s$. Using (6) and (3), the closed loop teleoperators can be represented as

$$
\begin{aligned}
\ddot{q}_{m}= & M_{m}^{-1}\left(q_{m}\right)\left[K_{P m} q_{s}\left(t-\gamma_{d}(t)\right)-K_{P m} q_{m}-K_{D m} \dot{q}_{m}-\right. \\
& C_{m}\left(q_{m}, \dot{q}_{m}\right) \dot{q}_{m}+\hat{G}_{m}\left(q_{m}\right)-G_{m}\left(q_{m}\right)-S_{m} q_{m}- \\
& \left.D_{m} \dot{q}_{m}\right] \\
\ddot{q}_{s}= & M_{s}^{-1}\left(q_{s}\right)\left[K_{P s} q_{m}\left(t-\gamma_{d}(t)\right)-K_{P s} q_{s}-K_{D s} \dot{q}_{s}-\right. \\
& \left.C_{s}\left(q_{s}, \dot{q}_{s}\right) \dot{q}_{s}+\hat{G}_{s}\left(q_{s}\right)-G_{s}\left(q_{s}\right)-S_{s} q_{s}-D_{s} \dot{q}_{s}\right] .
\end{aligned}
$$

To derive the stability condition for the systems (8) and (9), we consider the following Lyapunov-Krasovskii functional

$$
V=V_{1}+V_{2}+V_{3}+V_{4}
$$

with

$$
\begin{aligned}
V_{1}= & \dot{q}_{m}^{\mathrm{T}} M_{m}\left(q_{m}\right) \dot{q}_{m}+\dot{q}_{s}^{\mathrm{T}} M_{s}\left(q_{s}\right) \dot{q}_{s} \\
V_{2}= & \Lambda_{d} \int_{\left(t-\gamma_{d}(t)\right)}^{t} \dot{q}_{m}^{\mathrm{T}}(\eta) \dot{q}_{m}(\eta) \mathrm{d} \eta+ \\
& \Lambda_{d} \int_{\left(t-\gamma_{d}(t)\right)}^{t} \dot{q}_{s}^{\mathrm{T}}(\eta) \dot{q}_{s}(\eta) \mathrm{d} \eta \\
V_{3}= & K_{P m}\left(q_{m}-q_{s}\right)^{\mathrm{T}}\left(q_{m}-q_{s}\right)+S_{m} q_{m}^{\mathrm{T}} q_{m}+S_{s} q_{s}^{\mathrm{T}} q_{s} \\
V_{4}= & \tilde{\theta}_{m}^{\mathrm{T}} \Gamma_{m}^{-1} \tilde{\theta}_{m}+\tilde{\theta}_{s}^{\mathrm{T}} \Gamma_{s}^{-1} \tilde{\theta}_{s}
\end{aligned}
$$

where $\Lambda_{d}=\frac{1}{1-\left|\dot{\gamma}_{d}(t)\right|}$ with $\left|\dot{\gamma}_{d}(t)\right| \leqslant \varsigma \prec 1$. We now take the derivative (10) along the trajectories of the closed loop teleoperation systems (8), (9). Then, we apply Property 1 , adaptive control laws $\dot{\tilde{\theta}}_{m}$ and $\dot{\tilde{\theta}}_{s}$ along with projection mech- anism (7), $K_{P m}=K_{P s}$ and the following inequalities

$$
\begin{aligned}
& 2 \dot{q}_{m}^{\mathrm{T}} \int_{\left(t-\gamma_{d}(t)\right)}^{t} \dot{q}_{s}^{\mathrm{T}}(\eta) \mathrm{d} \eta \leqslant \dot{q}_{m}^{\mathrm{T}} \dot{q}_{m}+\int_{\left(t-\gamma_{d}(t)\right)}^{t} \dot{q}_{s}^{\mathrm{T}}(\eta) \dot{q}_{s}(\eta) \mathrm{d} \eta \\
& 2 \dot{q}_{s}^{\mathrm{T}} \int_{\left(t-\gamma_{d}(t)\right)}^{t} \dot{q}_{m}^{\mathrm{T}}(\eta) \mathrm{d} \eta \leqslant \dot{q}_{s}^{\mathrm{T}} \dot{q}_{s}+\int_{\left(t-\gamma_{d}(t)\right)}^{t} \dot{q}_{m}^{\mathrm{T}}(\eta) \dot{q}_{m}(\eta) \mathrm{d} \eta .
\end{aligned}
$$

$\dot{V}$ can be simplified as

$$
\begin{gathered}
\dot{V} \leqslant-\mathcal{K}_{m} \dot{q}_{m}^{\mathrm{T}} \dot{q}_{m}-\mathcal{L}_{s} \dot{q}_{s}^{\mathrm{T}} \dot{q}_{s}-\sigma \dot{q}_{s}^{\mathrm{T}}\left(t-\gamma_{d}(t)\right) \times \\
\dot{q}_{s}\left(t-\gamma_{d}(t)\right)-\sigma \dot{q}_{m}^{\mathrm{T}}\left(t-\gamma_{d}(t)\right) \dot{q}_{m}\left(t-\gamma_{d}(t)\right)+ \\
K_{P m} \int_{\left(t-\gamma_{d}(t)\right)}^{t} \dot{q}_{s}^{\mathrm{T}}(\eta) \dot{q}_{s}(\eta) \mathrm{d} \eta+K_{P m} \times \\
\int_{\left(t-\gamma_{d}(t)\right)}^{t} \dot{q}_{m}^{\mathrm{T}}(\eta) \dot{q}_{m}(\eta) \mathrm{d} \eta
\end{gathered}
$$

where $\mathcal{K}_{m}=\left(2 K_{D m}+2 D_{m}-\Lambda_{d}-K_{P m}\right), \quad \mathcal{L}_{s}=$ $\left(2 K_{D s}+2 D_{s}-\Lambda_{d}-K_{P m}\right)$ and $\sigma=\Lambda_{d}(1-\varsigma)$. Let us now first apply Schwartz inequality on the last two terms and then use the bound on the time varying delays. Then, we can write $\dot{V}$ in the following simplified form

$$
\begin{aligned}
\dot{V} \leqslant & -\mathcal{K}_{m} \dot{q}_{m}^{\mathrm{T}} \dot{q}_{m}-\mathcal{L}_{s} \dot{q}_{s}^{\mathrm{T}} \dot{q}_{s}-\sigma \dot{q}_{s}^{\mathrm{T}}\left(t-\gamma_{d}(t)\right) \dot{q}_{s}\left(t-\gamma_{d}(t)\right)- \\
& \sigma \dot{q}_{m}^{\mathrm{T}}\left(t-\gamma_{d}(t)\right) \dot{q}_{m}\left(t-\gamma_{d}(t)\right)+K_{P m} \mathcal{A}_{\alpha} \times \\
& \int_{\left(t-\gamma_{d}(t)\right)}^{t} \dot{q}_{m}(\eta)^{\mathrm{T}} \dot{q}_{m}(\eta) \mathrm{d} \eta+K_{P m} \mathcal{A}_{\alpha} \times \\
& \int_{\left(t-\gamma_{d}(t)\right)}^{t} \dot{q}_{s}(\eta)^{\mathrm{T}} \dot{q}_{s}(\eta) \mathrm{d} \eta
\end{aligned}
$$

where $\left|\gamma_{d}\right| \leqslant \mathcal{A}_{\alpha}$. Taking the integral from zero to time $T$, $\dot{V}$ leads to

$$
\begin{array}{r}
V(T)-V(0) \leqslant-\mathcal{K}_{m} \int_{0}^{T} \dot{q}_{m}^{\mathrm{T}} \dot{q}_{m} \mathrm{~d} t-\mathcal{L}_{s} \int_{0}^{T} \dot{q}_{s}^{\mathrm{T}} \dot{q}_{s} \mathrm{~d} t- \\
\sigma \int_{0}^{T} \dot{q}_{s}^{\mathrm{T}}\left(t-\gamma_{d}(t)\right) \dot{q}_{s}\left(t-\gamma_{d}(t)\right) \mathrm{d} t-
\end{array}
$$




$$
\begin{aligned}
& \sigma \int_{0}^{T} \dot{q}_{m}^{\mathrm{T}}\left(t-\gamma_{d}(t)\right) \dot{q}_{m}\left(t-\gamma_{d}(t)\right) \mathrm{d} t+ \\
& K_{P m} \mathcal{A}_{\alpha} \int_{0}^{T} \int_{\left(t-\gamma_{d}(t)\right)}^{t} \dot{q}_{m}(\eta)^{\mathrm{T}} \dot{q}_{m}(\eta) \mathrm{d} \eta \mathrm{d} t+ \\
& K_{P m} \mathcal{A}_{\alpha} \int_{0}^{T} \int_{\left(t-\gamma_{d}(t)\right)}^{t} \dot{q}_{s}(\eta)^{\mathrm{T}} \dot{q}_{s}(\eta) \mathrm{d} \eta \mathrm{d} t
\end{aligned}
$$

We again apply Schwartz inequality for the last two terms and then the bound on $\dot{V}$ can be calculated as $V(T)-V(0) \leqslant-\eta_{m}\left\|\dot{q}_{m}\right\|_{2}^{2}-\eta_{s}\left\|\dot{q}_{s}\right\|_{2}^{2}-\sigma \| \dot{q}_{m}(t-$ $\left.\gamma_{d}(t)\right)\left\|_{2}^{2}-\sigma\right\| \dot{q}_{s}\left(t-\gamma_{d}(t) \|_{2}^{2}\right.$ with $\eta_{m}=\left(\mathcal{K}_{m}-K_{P m} \mathcal{A}_{\alpha}^{2}\right)$ and $\eta_{s}=\left(\mathcal{L}_{s}-K_{P m} \mathcal{A}_{\alpha}^{2}\right)$. This implies that $\left(\dot{q}_{m}, \dot{q}_{s}, \dot{q}_{m}(t-\right.$ $\left.\left.\gamma_{d}(t)\right), \dot{q}_{s}\left(t-\gamma_{d}(t)\right)\right) \in \mathcal{L}_{2}$ and $\left(\dot{q}_{m}, \dot{q}_{s}, \dot{q}_{m}\left(t-\gamma_{d}(t)\right), \dot{q}_{s}(t-\right.$ $\left.\left.\gamma_{d}(t)\right), \dot{q}_{m}-\dot{q}_{s}, \dot{q}_{s}-\dot{q}_{m}, q_{m}, q_{s}, q_{m}-q_{s}, q_{s}-q_{m}\right) \in \mathcal{L}_{\infty}$. To show the bound on the two terms $\left(q_{m}-q_{s}\left(t-\gamma_{d}(t)\right)\right)$ and $\left(q_{s}-q_{m}\left(t-\gamma_{d}(t)\right)\right)$, let's first write them as

$$
\begin{array}{r}
\left(q_{m}-q_{s}\right)+\int_{\left(t-\gamma_{d}(t)\right)}^{t} \dot{q}_{s}(\eta) \mathrm{d} \eta \\
\left(q_{s}-q_{m}\right)+\int_{\left(t-\gamma_{d}(t)\right)}^{t} \dot{q}_{m}(\eta) \mathrm{d} \eta .
\end{array}
$$

Using Schwartz inequality, the bound for the terms $\int_{\left(t-\gamma_{d}(t)\right)}^{t} \dot{q}_{m}(\eta) \mathrm{d} \eta$ and $\int_{\left(t-\gamma_{d}(t)\right)}^{t} \dot{q}_{s}(\eta) \mathrm{d} \eta$ can be manipulated as $\mathcal{A}_{\alpha}^{\frac{1}{2}}\left\|\dot{q}_{m}\right\|_{2}$ and $\mathcal{A}_{\alpha}^{\frac{1}{2}}\left\|\dot{q}_{s}\right\|_{2}$. Since $q_{m}$ and $q_{s}$ are bounded, then we have $\left(q_{m}-q_{s}\left(t-\gamma_{d}(t)\right)\right) \in \mathcal{L}_{\infty}$ and $\left(q_{s}-q_{m}\left(t-\gamma_{d}(t)\right)\right) \in \mathcal{L}_{\infty}$. In view of our above analysis, Property 2, Property 3 , the boundedness property of the parameter estimates from projection mechanism (7) and invariance theorem ${ }^{[26]}$, we can conclude that, for the given $\varsigma$, there exists $K_{P m}=K_{P s}, K_{D m}$ and $K_{D s}$ such that the position, velocity and tracking error between master and slave manipulators in (8), (9) with passive input (4), (5) under symmetrical time varying delays are asymptotically stable.

We now design bilateral teleoperation systems where the forward and backward data packet delays are not symmetrical nature as $\gamma_{d m}(t) \neq \gamma_{d s}(t)$. The input algorithms for the systems (3) are designed as

$$
\begin{aligned}
\tau_{m} & =-K_{P m}\left(q_{m}-q_{s}\left(t-\gamma_{d s}(t)\right)\right)-K_{D m} \dot{q}_{m}+\hat{G}_{m}\left(q_{m}\right) \\
\tau_{s} & =K_{P s}\left(q_{m}\left(t-\gamma_{d m}(t)\right)-q_{s}\right)-K_{D s} \dot{q}_{s}+\hat{G}_{s}\left(q_{s}\right) .
\end{aligned}
$$

The control scheme (19) is shown in Fig. 1 with $\gamma_{d m}(t) \neq$ $\gamma_{d s}(t)$. Using (3) and (19), the closed loop teleoperators under unsymmetrical time varying delays can be derived as

$$
\begin{aligned}
\ddot{q}_{m}= & M_{m}^{-1}\left(q_{m}\right)\left[K_{P m} q_{s}\left(t-\gamma_{d s}(t)\right)-K_{P m} q_{m}-K_{D m} \dot{q}_{m}-\right. \\
& C_{m}\left(q_{m}, \dot{q}_{m}\right) \dot{q}_{m}+\hat{G}_{m}\left(q_{m}\right)-G_{m}\left(q_{m}\right)-S_{m} q_{m}- \\
& \left.D_{m} \dot{q}_{m}\right] \\
\ddot{q}_{s}= & M_{s}^{-1}\left(q_{s}\right)\left[K_{P s} q_{m}\left(t-\gamma_{d m}(t)\right)-K_{P s} q_{s}-K_{D s} \dot{q}_{s}-\right. \\
& \left.C_{s}\left(q_{s}, \dot{q}_{s}\right) \dot{q}_{s}+\hat{G}_{s}\left(q_{s}\right)-G_{s}\left(q_{s}\right)-S_{s} q_{s}-D_{s} \dot{q}_{s}\right] .
\end{aligned}
$$

To analyze the convergence property of the teleoperators (20), let us use the following Lyapunov-Krasovskii function

$$
V=V_{1}+V_{2}+V_{3}+V_{4}
$$

where

$$
\begin{aligned}
V_{1}= & \dot{q}_{m}^{\mathrm{T}} M_{m}\left(q_{m}\right) \dot{q}_{m}+\dot{q}_{s}^{\mathrm{T}} M_{s}\left(q_{s}\right) \dot{q}_{s} \\
V_{2}= & \Lambda_{d m} \int_{\left(t-\gamma_{d m}(t)\right)}^{t} \dot{q}_{m}^{\mathrm{T}}(\eta) \dot{q}_{m}(\eta) \mathrm{d} \eta+ \\
& \Lambda_{d s} \int_{\left(t-\gamma_{d s}(t)\right)}^{t} \dot{q}_{s}^{\mathrm{T}}(\eta) \dot{q}_{s}(\eta) \mathrm{d} \eta \\
V_{3}= & K_{P m}\left(q_{m}-q_{s}\right)^{\mathrm{T}}\left(q_{m}-q_{s}\right)+S_{m} q_{m}^{\mathrm{T}} q_{m}+S_{s} q_{s}^{\mathrm{T}} q_{s} \\
V_{4}= & \tilde{\theta}_{m}^{\mathrm{T}} \Gamma_{m}^{-1} \tilde{\theta}_{m}+\tilde{\theta}_{s}^{\mathrm{T}} \Gamma_{s}^{-1} \tilde{\theta}_{s}
\end{aligned}
$$

where $\Lambda_{d m}=\frac{1}{1-\left|\dot{\gamma}_{d m}(t)\right|}$ and $\Lambda_{d s}=\frac{1}{1-\left|\dot{\gamma}_{d s}(t)\right|}$ with $\left|\dot{\gamma}_{d m}(t)\right| \leqslant \varsigma_{d m}<1$ and $\left|\dot{\gamma}_{d s}(t)\right| \leqslant \varsigma_{d s}<1$. Using the Property $1,(20)$, adaptation laws with projection mechanism (7), $K_{P m}=K_{P s}$ and the following inequalities

$$
\begin{gathered}
2 \dot{q}_{m}^{\mathrm{T}} \int_{\left(t-\gamma_{d s}(t)\right)}^{t} \dot{q}_{s}^{\mathrm{T}}(\eta) \mathrm{d} \eta \leqslant \dot{q}_{m}^{\mathrm{T}} \dot{q}_{m}+\int_{\left(t-\gamma_{d s}(t)\right)}^{t} \dot{q}_{s}^{\mathrm{T}}(\eta) \dot{q}_{s}(\eta) \mathrm{d} \eta \\
2 \dot{q}_{s}^{\mathrm{T}} \int_{\left(t-\gamma_{d m}(t)\right)}^{t} \dot{q}_{m}^{\mathrm{T}}(\eta) \mathrm{d} \eta \leqslant \dot{q}_{s}^{\mathrm{T}} \dot{q}_{s}+\int_{\left(t-\gamma_{d m}(t)\right)}^{t} \dot{q}_{m}^{\mathrm{T}}(\eta) \dot{q}_{m}(\eta) \mathrm{d} \eta .
\end{gathered}
$$

$\dot{V}$ can be represented as

$$
\begin{aligned}
\dot{V} \leqslant-\mathcal{K}_{m u} \dot{q}_{m}^{\mathrm{T}} \dot{q}_{m}-\mathcal{L}_{s u} \dot{q}_{s}^{\mathrm{T}} \dot{q}_{s}-\sigma_{m s} \dot{q}_{s}^{\mathrm{T}}\left(t-\gamma_{d s}(t)\right) \times \\
\left.\dot{q}_{s}\left(t-\gamma_{d s}(t)\right)-\sigma_{m u} \dot{q}_{m}^{\mathrm{T}}\left(t-\gamma_{d m}(t)\right)\right) \dot{q}_{m}\left(t-\gamma_{d m}(t)\right)+ \\
\int_{\left(t-\gamma_{d s}(t)\right)}^{t} \dot{q}_{s}^{\mathrm{T}}(\eta) \dot{q}_{s}(\eta) \mathrm{d} \eta+\int_{\left(t-\gamma_{d m}(t)\right)}^{t} \dot{q}_{m}^{\mathrm{T}}(\eta) \dot{q}_{m}(\eta) \mathrm{d} \eta
\end{aligned}
$$

where $\mathcal{K}_{m u}=\left(2 K_{D m}+2 D_{m}-\Lambda_{d m}-K_{P m}\right), \quad \mathcal{L}_{s u}=$ $\left(2 K_{D s}+2 D_{s}-\Lambda_{d s}-K_{P m}\right), \quad \sigma_{m s}=\Lambda_{d s}\left(1-\varsigma_{d s}\right)$ and $\sigma_{m u}=\Lambda_{d m}\left(1-\varsigma_{d m}\right)$. We now use Schwartz inequality on the last two terms and then take integral from zero to time $t=T$. Then, $\dot{V}$ can be written as

$$
\begin{aligned}
V(T)- & V(0) \leqslant-\mathcal{K}_{m u} \int_{0}^{T} \dot{q}_{m}^{\mathrm{T}} \dot{q}_{m} \mathrm{~d} t-\mathcal{L}_{s u} \int_{0}^{T} \dot{q}_{s}^{\mathrm{T}} \dot{q}_{s} \mathrm{~d} t- \\
& \sigma_{m s} \int_{0}^{T} \dot{q}_{s}^{\mathrm{T}}\left(t-\gamma_{d s}(t)\right) \dot{q}_{s}\left(t-\gamma_{d s}(t)\right) \mathrm{d} t-\sigma_{m u} \times \\
& \int_{0}^{T} \dot{q}_{m}^{\mathrm{T}}\left(t-\gamma_{d m}(t)\right) \dot{q}_{m}\left(t-\gamma_{d m}(t)\right) \mathrm{d} t+K_{P m} \mathcal{A}_{d \alpha} \times \\
& \int_{0}^{T} \int_{\left(t-\gamma_{d m}(t)\right)}^{t} \dot{q}_{m}(\eta)^{\mathrm{T}} \dot{q}_{m}(\eta) \mathrm{d} \eta \mathrm{d} t+K_{P m} \mathcal{A}_{s \alpha} \times \\
& \int_{0}^{T} \int_{\left(t-\gamma_{d s}(t)\right)}^{t} \dot{q}_{s}(\eta)^{\mathrm{T}} \dot{q}_{s}(\eta) \mathrm{d} \eta \mathrm{d} t
\end{aligned}
$$

with $\left|\gamma_{d m}\right| \leqslant \mathcal{A}_{d \alpha}$ and $\left|\gamma_{d s}\right| \leqslant \mathcal{A}_{s \alpha}$. Applying Schwartz inequality on the last two terms, $\dot{V}$ can be simplified further

$$
\begin{aligned}
V(T)-V(0) \leqslant & -\eta_{m u}\left\|\dot{q}_{m}\right\|_{2}^{2}-\sigma_{m u}\left\|\dot{q}_{m}\left(t-\gamma_{d m}(t)\right)\right\|_{2}^{2}- \\
& \eta_{s u}\left\|\dot{q}_{s}\right\|_{2}^{2}-\sigma_{m s} \| \dot{q}_{s}\left(t-\gamma_{d s}(t) \|_{2}^{2}\right.
\end{aligned}
$$

where $\eta_{m u}=\left(\mathcal{K}_{m u}-K_{P m} \mathcal{A}_{d \alpha}^{2}\right)$ and $\eta_{s u}=\left(\mathcal{L}_{s u}-K_{P m} \mathcal{A}_{s \alpha}^{2}\right)$. This implies that $\left(\dot{q}_{m}, \dot{q}_{s}, \dot{q}_{m}\left(t-\gamma_{d m}(t)\right), \dot{q}_{s}\left(t-\gamma_{d s}(t)\right)\right) \in$ $\mathcal{L}_{2}$ and $\left(\dot{q}_{m}, \dot{q}_{s}, \dot{q}_{m}\left(t-\gamma_{d m}(t)\right), \dot{q}_{s}\left(t-\gamma_{d s}(t)\right)\right) \in \mathcal{L}_{\infty}$. We can also show the bounds on the terms $\left(q_{m}-q_{s}\left(t-\gamma_{d s}(t)\right)\right)$ and $\left(q_{s}-q_{m}\left(t-\gamma_{d m}(t)\right)\right)$. To show that, we first rewrite the term $\left(q_{m}-q_{s}\left(t-\gamma_{d s}(t)\right)\right)$ as

$$
\left(q_{m}-q_{s}\right)+\int_{\left(t-\gamma_{d s}(t)\right)}^{t} \dot{q}_{s}(\eta) \mathrm{d} \eta
$$


and the term $\left(q_{s}-q_{m}\left(t-\gamma_{d m}(t)\right)\right)$ as

$$
\left(q_{s}-q_{m}\right)+\int_{\left(t-\gamma_{d m}(t)\right)}^{t} \dot{q}_{m}(\eta) \mathrm{d} \eta .
$$

In view of Schwartz inequality, we can obtain the bound on $\int_{\left(t-\gamma_{d m}(t)\right)}^{t} \dot{q}_{m}(\eta) \mathrm{d} \eta$ and $\int_{\left(t-\gamma_{d s}(t)\right)}^{t} \dot{q}_{s}(\eta) \mathrm{d} \eta$ as $\mathcal{A}_{d \alpha}^{\frac{1}{2}}\left\|\dot{q}_{m}\right\|_{2}$ and $\mathcal{A}_{s \alpha}^{\frac{1}{2}}\left\|\dot{q}_{s}\right\|_{2}$ imply the bound $\left(q_{m}-q_{s}\left(t-\gamma_{d s}(t)\right)\right) \in \mathcal{L}_{\infty}$ and $\left(q_{s}-q_{m}\left(t-\gamma_{d m}(t)\right)\right) \in \mathcal{L}_{\infty}$. Then, applying invariance theorem ${ }^{[26]}$, Property 2 and Property 3 along with the parameter projection mechanism (7), we can conclude that the position, velocity and tracking error between master and slave manipulators converge to zero as the time goes to infinity.

\section{Teleoperation system with delayed position and velocity signals}

Let us now design teleoperation systems by coupling local and remote sites by delaying position and velocity signals of the master and slave systems. The input algorithms for the systems (3) with symmetrical time varying delays can be designed as follows

$$
\begin{aligned}
\tau_{m}= & -K_{D m}\left(\dot{q}_{m}-\dot{q}_{s}\left(t-\gamma_{d}(t)\right)\right)+\hat{G}_{m}\left(q_{m}\right)- \\
& K_{P m}\left(q_{m}-q_{s}\left(t-\gamma_{d}(t)\right)\right)-\zeta_{m} \dot{q}_{m} \\
\tau_{s}= & K_{D s}\left(\dot{q}_{m}\left(t-\gamma_{d}(t)\right)-\dot{q}_{s}\right)-K_{P s} q_{s}+\hat{G}_{s}\left(q_{s}\right)- \\
& K_{P s}\left(q_{s}-q_{m}\left(t-\gamma_{d}(t)\right)\right)-\zeta_{s} \dot{q}_{s}
\end{aligned}
$$

with $\zeta_{m}>0$ and $\zeta_{s}>0$. The implementation block diagram of the control scheme (26) is presented in Fig. 2 with $\gamma_{d m}(t)=\gamma_{d s}(t)=\gamma_{d}(t)$. The closed loop teleoperation systems with input algorithms (26) can be formulated as

$$
\begin{aligned}
\ddot{q}_{m}= & M_{m}^{-1}\left(q_{m}\right)\left[K_{D m} \dot{q}_{s}\left(t-\gamma_{d}(t)\right)-K_{P m} q_{m}-K_{D m} \dot{q}_{m}-\right. \\
& C_{m}\left(q_{m}, \dot{q}_{m}\right) \dot{q}_{m}+\hat{G}_{m}\left(q_{m}\right)-G_{m}\left(q_{m}\right)-S_{m} q_{m}- \\
& \left.D_{m} \dot{q}_{m}+K_{P m} q_{s}\left(t-\gamma_{d}(t)\right)-\zeta_{m} \dot{q}_{m}\right] \\
\ddot{q}_{s}= & M_{s}^{-1}\left(q_{s}\right)\left[K_{D s} \dot{q}_{m}\left(t-\gamma_{d}(t)\right)-K_{D s} \dot{q}_{s}-K_{P s} q_{s}-\right. \\
& C_{s}\left(q_{s}, \dot{q}_{s}\right) \dot{q}_{s}+\hat{G}_{s}\left(q_{s}\right)-G_{s}\left(q_{s}\right)-S_{s} q_{s}-D_{s} \dot{q}_{s}+ \\
& \left.K_{P s} q_{m}\left(t-\gamma_{d}(t)\right)-\zeta_{s} \dot{q}_{s}\right] .
\end{aligned}
$$

For the convergence analysis of the teleoperation systems (27), (28), let us employ the following Lyapunov-Krasovskii function

$$
V=V_{1}+V_{2}+V_{3}+V_{4}
$$

with

$$
\begin{aligned}
V_{1}= & \dot{q}_{m}^{\mathrm{T}} M_{m}\left(q_{m}\right) \dot{q}_{m}+\dot{q}_{s}^{\mathrm{T}} M_{s}\left(q_{s}\right) \dot{q}_{s} \\
V_{2}= & \Lambda_{d 1} \int_{\left(t-\gamma_{d}(t)\right)}^{t} \dot{q}_{m}^{\mathrm{T}}(\eta) \dot{q}_{m}(\eta) \mathrm{d} \eta+\Lambda_{d 2} \int_{\left(t-\gamma_{d}(t)\right)}^{t} \dot{q}_{s}^{\mathrm{T}}(\eta) \\
& \dot{q}_{s}(\eta) \mathrm{d} \eta \\
V_{3}= & K_{P m}\left(q_{m}-q_{s}\right)^{\mathrm{T}}\left(q_{m}-q_{s}\right)+S_{m} q_{m}^{\mathrm{T}} q_{m}+S_{s} q_{s}^{\mathrm{T}} q_{s} \\
V_{4}= & \tilde{\theta}_{m}^{\mathrm{T}} \Gamma_{m}^{-1} \tilde{\theta}_{m}+\tilde{\theta}_{s}^{\mathrm{T}} \Gamma_{s}^{-1} \tilde{\theta}_{s}
\end{aligned}
$$

where $\Lambda_{d 1}=\frac{K_{D m}}{\left.1-\mid \dot{\gamma}_{d}(t)\right) \mid}$ and $\Lambda_{d 2}=\frac{K_{D s}}{\left.1-\mid \dot{\gamma}_{d}(t)\right) \mid}$. Using $K_{P m}=$ $K_{P s}, K_{D m}=K_{D s}$, adaptive control with projection mechanism (7), Property 1, (12) and

$$
\begin{aligned}
2 \dot{q}_{m}^{\mathrm{T}} \dot{q}_{s}\left(t-\gamma_{d}(t)\right) & \leqslant \dot{q}_{m}^{\mathrm{T}} \dot{q}_{m}+\dot{q}_{s}^{\mathrm{T}}\left(t-\gamma_{d}(t)\right) \dot{q}_{s}^{\mathrm{T}}\left(t-\gamma_{d}(t)\right) \\
2 \dot{q}_{s}^{\mathrm{T}} \dot{q}_{m}\left(t-\gamma_{d}(t)\right) & \leqslant \dot{q}_{s}^{\mathrm{T}} \dot{q}_{s}+\dot{q}_{m}^{\mathrm{T}}\left(t-\gamma_{d}(t)\right) \dot{q}_{m}\left(t-\gamma_{d}(t)\right) .
\end{aligned}
$$

The time derivative of (29) along the trajectories of (27), (28) can be simplified as $V(T)-V(0) \leqslant$ $-\pi_{D m}\left\|\dot{q}_{m}\right\|_{2}^{2}-\pi_{D s}\left\|\dot{q}_{s}\right\|_{2}$ with $\pi_{D m}=\left(K_{D m}+2 D_{m}+\right.$ $\left.K_{P m} \mathcal{A}_{\alpha}^{2}+\zeta_{m}-\Lambda_{d 1}-K_{P m}\right)$ and $\pi_{D s}=\left(K_{D m}+2 D_{s}+\right.$ $\left.K_{P m} \mathcal{A}_{\alpha}^{2}-\Lambda_{d 2}-K_{P m}\right)$. This implies that $\left(\dot{q}_{m}, \dot{q}_{s}\right) \in$ $\mathcal{L}_{2}$ and $\left(q_{m}, q_{s}, \dot{q}_{m}, \dot{q}_{s}, \dot{q}_{m}-\dot{q}_{s}, \dot{q}_{s}-\dot{q}_{m}, q_{m}-q_{s}, q_{s}-q_{m}\right) \in$ $\mathcal{L}_{\infty}$. Now, we need to show the bounds on the terms $\left(\dot{q}_{m}-\dot{q}_{s}\left(t-\gamma_{d}(t)\right)\right)$ and $\left(\dot{q}_{s}-\dot{q}_{m}\left(t-\gamma_{d}(t)\right)\right)$ from the following relationship

$$
\begin{array}{r}
\left(\dot{q}_{m}-\dot{q}_{s}\right)+\int_{\left(t-\gamma_{d}(t)\right)}^{t} \ddot{q}_{s}(\eta) \mathrm{d} \eta \\
\left(\dot{q}_{s}-\dot{q}_{m}\right)+\int_{\left(t-\gamma_{d}(t)\right)}^{t} \ddot{q}_{m}(\eta) \mathrm{d} \eta .
\end{array}
$$


Fig. 2 Control scheme for bilateral teleoperation systems with delayed position and velocity signals for symmetrical time varying delay $\gamma_{d m}(t)=\gamma_{d s}(t)=\gamma_{d}(t)$ and unsymmetrical time varying delay $\gamma_{d m}(t) \neq \gamma_{d s}(t)$ 
Applying Schwartz inequality, we can write $\int_{\left(t-\gamma_{d}(t)\right)}^{t} \ddot{q}_{m}(\eta) \mathrm{d} \eta$ and $\int_{\left(t-\gamma_{d}(t)\right)}^{t} \ddot{q}_{s}(\eta) \mathrm{d} \eta$ as $\mathcal{A}_{\alpha}^{\frac{1}{2}}\left\|\ddot{q}_{m}\right\|_{2}$ and $\mathcal{A}_{\alpha}^{\frac{1}{2}}\left\|\ddot{q}_{s}\right\|_{2}$. This implies that $\left(\dot{q}_{m}-\dot{q}_{s}\left(t-\gamma_{d}(t)\right)\right) \in \mathcal{L}_{\infty}$ and $\left(\dot{q}_{s}-\dot{q}_{m}\left(t-\gamma_{d}(t)\right)\right) \in \mathcal{L}_{\infty}$. From (17) and (18), we also have $\left(q_{m}-q_{s}\left(t-\gamma_{d}(t)\right)\right) \in \mathcal{L}_{\infty}$ and $\left(q_{m}-q_{s}\left(t-\gamma_{d}(t)\right)\right) \in$ $\mathcal{L}_{\infty}$. Then, in view of Property 2, Property 3, projection mechanism for the parameter estimates (7) and invariance principle $^{[26]}$, we can conclude that the position, velocity and tracking error signals in the closed loop teleoperators (27), (28) are bounded and asymptotically converge to zero.

Finally, we design teleoperation systems by delaying position and velocity signals with unsymmetrical time varying communication delays as

$$
\begin{aligned}
\tau_{m}= & -K_{D m}\left(\dot{q}_{m}-\dot{q}_{s}\left(t-\gamma_{d s}(t)\right)\right)-K_{P m} q_{m}+\hat{G}_{m}\left(q_{m}\right)+ \\
& K_{P m} q_{s}\left(t-\gamma_{d s}(t)\right)-\zeta_{m} \dot{q}_{m} \\
\tau_{s}= & K_{D s}\left(\dot{q}_{m}\left(t-\gamma_{d m}(t)\right)-\dot{q}_{s}\right)-K_{P s} q_{s}+\hat{G}_{s}\left(q_{s}\right)+ \\
& K_{P s} q_{m}\left(t-\gamma_{d m}(t)\right)-\zeta_{s} \dot{q}_{s} .
\end{aligned}
$$

The control scheme is shown in Fig. 2 with $\gamma_{d m}(t) \neq$ $\gamma_{d s}(t)$. Using (33) and (3), the closed loop teleoperation systems can be derived as

$$
\begin{aligned}
\ddot{q}_{m}= & M_{m}^{-1}\left(q_{m}\right)\left[K_{D m} \dot{q}_{s}\left(t-\gamma_{d s}(t)\right)-K_{P m} q_{m}-K_{D m} \dot{q}_{m}-\right. \\
& C_{m}\left(q_{m}, \dot{q}_{m}\right) \dot{q}_{m}+\hat{G}_{m}\left(q_{m}\right)-G_{m}\left(q_{m}\right)-S_{m} q_{m}- \\
& \left.D_{m} \dot{q}_{m}+K_{P m} q_{s}\left(t-\gamma_{d s}(t)\right)-\zeta_{m} \dot{q}_{m}\right] \\
\ddot{q}_{s}= & M_{s}^{-1}\left(q_{s}\right)\left[K_{D s} \dot{q}_{m}\left(t-\gamma_{d m}(t)\right)-K_{D s} \dot{q}_{s}-K_{P s} q_{s}-\right. \\
& C_{s}\left(q_{s}, \dot{q}_{s}\right) \dot{q}_{s}+\hat{G}_{s}\left(q_{s}\right)-G_{s}\left(q_{s}\right)-S_{s} q_{s}-D_{s} \dot{q}_{s}+ \\
& \left.K_{P s} q_{m}\left(t-\gamma_{d m}(t)\right)-\zeta_{s} \dot{q}_{s}\right] .
\end{aligned}
$$

Now, for analyzing the stability of the closed loop systems (34), (35), we choose the following Lyapunov-Krasovskii functional

$$
V=V_{1}+V_{2}+V_{3}+V_{4}
$$

with

$$
\begin{aligned}
V_{1}= & \dot{q}_{m}^{\mathrm{T}} M_{m}\left(q_{m}\right) \dot{q}_{m}+\dot{q}_{s}^{\mathrm{T}} M_{s}\left(q_{s}\right) \dot{q}_{s} \\
V_{2}= & \Lambda_{d m 1} \int_{\left(t-\gamma_{d m}(t)\right)}^{t} \dot{q}_{m}^{\mathrm{T}}(\eta) \dot{q}_{m}(\eta) \mathrm{d} \eta+ \\
& \Lambda_{d s 2} \int_{\left(t-\gamma_{d s}(t)\right)}^{t} \dot{q}_{s}^{\mathrm{T}}(\eta) \dot{q}_{s}(\eta) \mathrm{d} \eta \\
V_{3}= & K_{P m}\left(q_{m}-q_{s}\right)^{\mathrm{T}}\left(q_{m}-q_{s}\right)+S_{m} q_{m}^{\mathrm{T}} q_{m}+S_{s} q_{s}^{\mathrm{T}} q_{s} \\
V_{4}= & \tilde{\theta}_{m}^{\mathrm{T}} \Gamma_{m}^{-1} \tilde{\theta}_{m}+\tilde{\theta}_{s}^{\mathrm{T}} \Gamma_{s}^{-1} \tilde{\theta}_{s}
\end{aligned}
$$

where $\Lambda_{d m 1}=\frac{K_{D m}}{1-\left|\dot{\gamma}_{d m}(t)\right|}$ and $\Lambda_{d s 2}=\frac{K_{D s}}{1-\left|\dot{\gamma}_{d s}(t)\right|}$. We now take the derivative (36) along the trajectories of the closed loop systems (34), (35). Then, using $K_{P m}=K_{P s}$, $K_{D m}=K_{D s}$, (22), adaptation laws with parameter projection scheme (7), Property 1 and

$$
\begin{aligned}
2 \dot{q}_{m}^{\mathrm{T}} \dot{q}_{s}\left(t-\gamma_{d s}(t)\right) & \leqslant \dot{q}_{m}^{\mathrm{T}} \dot{q}_{m}+\dot{q}_{s}^{\mathrm{T}}\left(t-\gamma_{d s}(t)\right) \dot{q}_{s}^{\mathrm{T}}\left(t-\gamma_{d s}(t)\right) \\
2 \dot{q}_{s}^{\mathrm{T}} \dot{q}_{m}\left(t-\gamma_{d m}(t)\right) & \leqslant \dot{q}_{s}^{\mathrm{T}} \dot{q}_{s}+\dot{q}_{m}^{\mathrm{T}}\left(t-\gamma_{d m}(t)\right) \dot{q}_{m}\left(t-\gamma_{d m}(t)\right) .
\end{aligned}
$$

We can simplify the time derivative of (36) as $V(T)-V(0) \leqslant-\xi_{s m}\left\|\dot{q}_{m}\right\|_{2}^{2}-\xi_{s s}\left\|\dot{q}_{s}\right\|_{2}$, where $\xi_{s m}=$ $\left(K_{D m}+2 D_{m}+K_{P m} \mathcal{A}_{d \alpha}^{2}+\zeta_{m}-\Lambda_{d m 1}\right), \quad$ and $\xi_{s s}=$
$\left(K_{D m}+2 D_{s}+K_{P m} \mathcal{A}_{s \alpha}^{2}+\zeta_{s}-\Lambda_{d s 2}\right)$. This means that $\left(\dot{q}_{m}, \dot{q}_{s}\right) \in \mathcal{L}_{2}$ and $\left(q_{m}, q_{s}, \dot{q}_{m}, \dot{q}_{s}, \dot{q}_{m}-\dot{q}_{s}, \dot{q}_{s}-\dot{q}_{m}, q_{m}-q_{s}\right.$, $\left.q_{s}-q_{m}\right) \in \mathcal{L}_{\infty}$. Using the relationship $\left(\dot{q}_{m}-\dot{q}_{s}\right)+$ $\int_{\left(t-\gamma_{d s}(t)\right)}^{t} \ddot{q}_{s}(\eta) \mathrm{d} \eta$ and $\left(\dot{q}_{s}-\dot{q}_{m}\right)+\int_{\left(t-\gamma_{d m}(t)\right)}^{t} \ddot{q}_{m}(\eta) \mathrm{d} \eta$, we can also show the bound on $\left(\dot{q}_{m}-\dot{q}_{s}\left(t-\gamma_{d s}(t)\right)\right) \in \mathcal{L}_{\infty}$ and $\left(\dot{q}_{s}-\dot{q}_{m}\left(t-\gamma_{d m}(t)\right)\right) \in \mathcal{L}_{\infty}$. Applying our previous analysis for (24), (25), it is also possible to guarantee $\left(q_{m}-q_{s}\left(t-\gamma_{d s}(t)\right)\right) \in \mathcal{L}_{\infty}$ and $\left(q_{s}-q_{m}\left(t-\gamma_{d s}(t)\right)\right) \in$ $\mathcal{L}_{\infty}$. Then, using invariance theorem ${ }^{[26]}$, Property 2, Property 3 and boundedness of parameter estimates from projection laws (7), we can state that the position, velocity, tracking error signals in (34) and (35) asymptotically convergent to zero.

\section{Evaluation results}

We now show the design and evaluation process of the proposed control methods on bilateral teleoperation systems constructed by a 2-DOF master and slave manipulator. For simplicity, we only present evaluation results of the teleoperation algorithms given in (19). In our evaluation, it is assumed that the local and remote sites are connected by Internet telecommunication channel. The motion dynamics of a 2-DOF master and slave manipulator can be written as

$$
\begin{aligned}
& M_{m}\left(q_{m}\right) \ddot{q}_{m}+C_{m}\left(q_{m}, \dot{q}_{m}\right) \dot{q}_{m}+G_{m}\left(q_{m}\right)=\tau_{m}+F_{h} \\
& M_{s}\left(q_{s}\right) \ddot{q}_{s}+C_{s}\left(q_{s}, \dot{q}_{s}\right) \dot{q}_{s}+G_{s}\left(q_{s}\right)=\tau_{s}-F_{e}
\end{aligned}
$$

where $M_{m}\left(q_{m}\right)=\left[\begin{array}{ll}m_{11} & m_{12} \\ m_{21} & m_{22}\end{array}\right], \quad C_{m}\left(q_{m}, \dot{q}_{m}\right)=$ $\left[\begin{array}{ll}c_{11} & c_{12} \\ c_{21} & c_{22}\end{array}\right], \quad F_{h}=\left[\begin{array}{l}F_{h 1} \\ F_{h 2}\end{array}\right], \quad F_{e}=\left[\begin{array}{l}F_{e 1} \\ F_{e 2}\end{array}\right]$ and $G_{m}\left(q_{m}\right)=\left[\begin{array}{l}G_{m}\left(q_{m 1}\right) \\ G_{m}\left(q_{m 2}\right)\end{array}\right]$ with $m_{11}=\left(2 l_{1} \cos q_{2}+l_{2}\right) l_{2} m_{2}+$ $l_{1}^{2}\left(m_{1}+m_{2}\right), m_{12}=\left(l_{2} m_{2}+l_{1} l_{2} m_{2} \cos q_{2}\right), m_{21}=\left(m_{2} l^{2}+\right.$ $\left.m_{2} l^{2} \cos q_{2}\right), m_{22}=m_{2} l_{2}^{2}, c_{11}=-l_{1} l_{2} m_{2} \sin q_{2} \dot{q}_{2}, c_{12}=$ $-l_{1} l_{2} m_{2} \sin q_{2}\left(\dot{q}_{2}+\dot{q}_{1}\right), \quad c_{21}=l_{1} l_{2} m_{2} \sin q_{2}, \quad c_{22}=0$, $G\left(q_{m 1}\right)=g m_{2} l_{2} \sin \left(q_{1}+q_{2}\right)+g\left(m_{1}+m_{2}\right) l_{1} \sin q_{1}$ and $G\left(q_{m 2}\right)=g m_{2} l_{2} \sin \left(q_{1}+q_{2}\right)$. The uncertain parameters for gravity loading vectors are chosen as $\theta_{m 1}=$ $m_{2} l_{2}, \theta_{m 2}=m_{1} l_{1}$ and $\theta_{m 3}=l_{1} m_{2}$. We also consider that $M_{s}\left(q_{s}\right)=M_{m}\left(q_{m}\right), C_{m}\left(q_{m}, \dot{q}_{m}\right)=C_{s}\left(q_{s}, \dot{q}_{s}\right) \dot{q}_{s}$ and $G_{m}\left(q_{m}\right)=G_{s}\left(q_{s}\right)$. The model parameters for master and slave manipulators are chosen as $m_{1}=15, m_{2}=5$, $l_{1}=1, l_{2}=0.5$ and $g=9.82$. In our first evaluation, the time varying delays in forward, $\gamma_{d m}(t)$, and backward, $\gamma_{d s}(t)$, internet telecommunication channels are assumed to be unsymmetrical nature as $\gamma_{d m}(t)=10+0.8 \sin (t)$ and $\gamma_{d s}(t)=5+0.5 \cos (t)$. The time history of the time varying delays are given in Fig. 3. The environment and human input forces are modeled as $F_{e 1}=S_{s 1} q_{s 1}+D_{s 1} \dot{q}_{s 1}$, $F_{e 2}=S_{s 2} q_{s 2}+D_{s 2} \dot{q}_{s 2}, F_{h 1}=k_{o 1}-S_{m 1} q_{m 1}-D_{m 1} \dot{q}_{m 1}$, $F_{h 2}=k_{o 2}-S_{m 2} q_{m 2}-D_{m 2} \dot{q}_{m 2}$ with $k_{o 1}>0$ and $k_{o 2}>0$.

In our evaluation, we consider three different types of interaction forces between slave and environment. In first case, the slave manipulators are operating in free space as $F_{e 1}=0$ and $F_{e 2}=0$. The parameters for human operator forces are chosen as $k_{o 1}=10, k_{o 2}=10, S_{m 1}=15$, $S_{m 2}=15, D_{m 1}=10$ and $D_{m 2}=20$. The control design parameters are selected as $\Gamma_{m}=I_{3 \times 3}, \Gamma_{s}=I_{3 \times 3}, K_{P m 1}=$ $K_{P s 1}=10, K_{P m 2}=K_{P s 2}=10, K_{D m 1}=K_{D m 2}=150$ and $K_{D s 1}=K_{D s 2}=150$. The evaluation results are presented 
in Fig. 4.

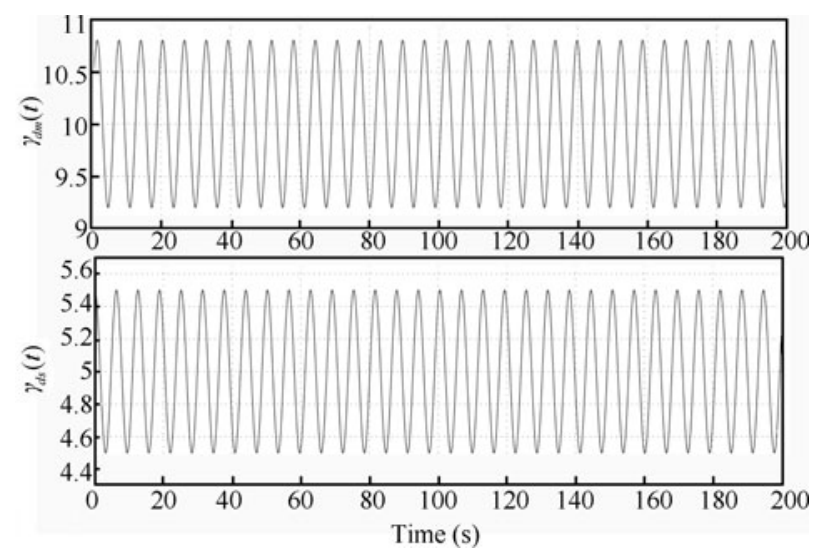

Fig. 3 Unsymmetrical forward $\gamma_{d m}(t)$ and backward $\gamma_{d s}(t)$ time varying communication delays in second
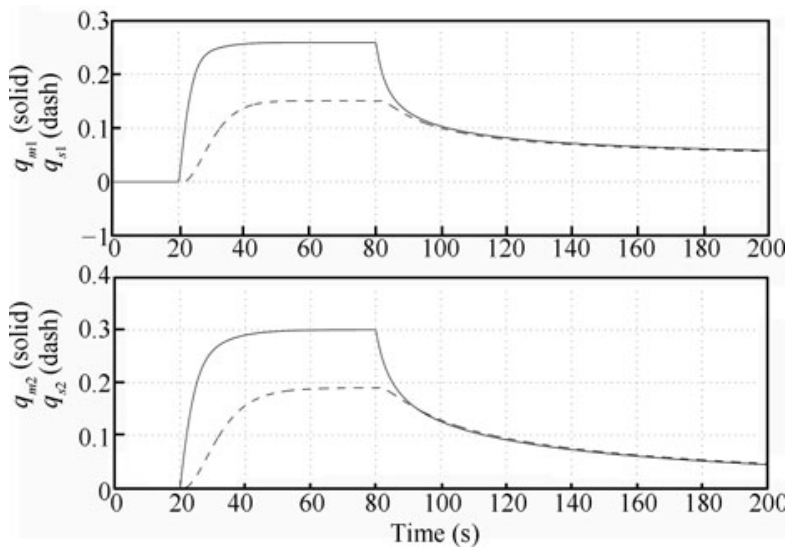

Fig. 4 Position tracking of the master and slave manipulators in radians with free remote environment space

Second, we consider that the remote slave manipulators are interacting with soft environment as $S_{s 1}=2, S_{s 2}=2$, $D_{s 1}=1$ and $D_{s 2}=1$. The parameters of the human input forces are chosen as $k_{o 1}=15, k_{o 2}=15, S_{m 1}=15$, $S_{m 2}=15, D_{m 1}=10$ and $D_{m 2}=20$. The control design parameters are chosen for this evaluation as $\Gamma_{m}=I_{3 \times 3}$, $\Gamma_{s}=I_{3 \times 3}, K_{P m 1}=K_{P s 1}=10, K_{P m 2}=K_{P s 2}=10$, $K_{D m 1}=K_{D m 2}=250, K_{D s 1}=K_{D s 2}=150$. The evaluation results are given in Fig. 5 with unsymmetrical time varying delays as depicted in Fig. 3.

In third case, we apply very hard interaction forces between remote slave manipulator and environment as $S_{s 1}=$ 50000, $S_{s 2}=50000, D_{s 1}=1$ and $D_{s 2}=1$. Then, we choose the parameters for human and master interaction forces as $k_{o 1}=15, k_{o 2}=15, S_{m 1}=15, S_{m 2}=15$, $D_{m 1}=10$ and $D_{m 2}=20$. The control design parameters are considered as $\Gamma_{m}=I_{3 \times 3}, \Gamma_{s}=I_{3 \times 3}, K_{P m 1}=$ $K_{P s 1}=10, K_{P m 2}=K_{P s 2}=10, K_{D m 1}=K_{D m 2}=250$, $K_{D s 1}=K_{D s 2}=150$. The unsymmetrical time varying delays are kept similar to our previous evaluation as depicted in Fig. 3. With these parameters, the design strategy proposed in (20) is applied to the given teleoperation systems. The evaluation results with very hard contact forces between slave and environment are shown in Fig. 6.

Let us now repeat the evaluation of third case with un- symmetrical random time varying delays. For our evaluation, the time derivative of the random time varying delays for the forward and backward communication channels are chosen as shown in Fig. 7. All other design parameters are kept similar to our previous case. The evaluation results are depicted in Fig. 8. The sampling time is chosen as $0.01 \mathrm{~s}$. From these results, we can see that the position and tracking error of the master and slave manipulators are asymptotically stable in the presence of unsymmetrical forward and backward time varying delays. The asymptotic stability is also guaranteed in the presence of free space, soft and very hard interaction forces between remote slave and environment.

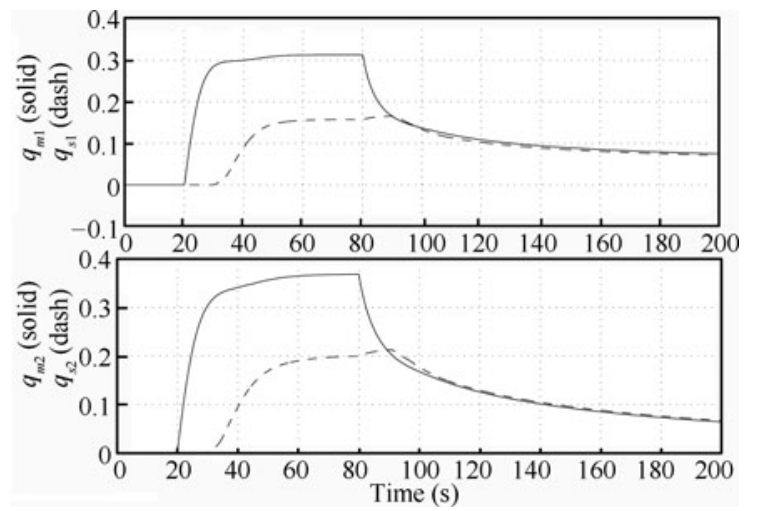

Fig. 5 Position tracking of the master and slave manipulator in radians with soft interaction between remote slave and environment



Fig. 6 Position tracking of the master and slave manipulator in radians with very hard interaction between remote slave and environment

Remark 1. We notice from our various evaluation results that the performance with the delayed positionvelocity signal based teleoperator provides better performance than the performance achieved under delayed position signal based teleoperator. This is mainly because of the fact that the position-velocity based teleoperator provides additional damping to the master and slave manipulator ensuring better coordination of the master and slave manipulator.

Remark 2. Note that the conditions for $K_{P m}=$ $K_{P s}$ can be removed by modifying $V_{1}$ and $V_{3}$ as $V_{1}=\dot{q}_{m}^{\mathrm{T}} M_{m}\left(q_{m}\right) \dot{q}_{m}+\frac{K_{P m}}{K_{P s}} \dot{q}_{s}^{\mathrm{T}} M_{s}\left(q_{s}\right) \dot{q}_{s}$ and $V_{3}=$ 
$K_{P m}\left(q_{m}-q_{s}\right)^{\mathrm{T}}\left(q_{m}-q_{s}\right)+P_{m} q_{m}^{\mathrm{T}} q_{m}+\frac{K_{P m}}{K_{P s}} P_{s} q_{s}^{\mathrm{T}} q_{s}$
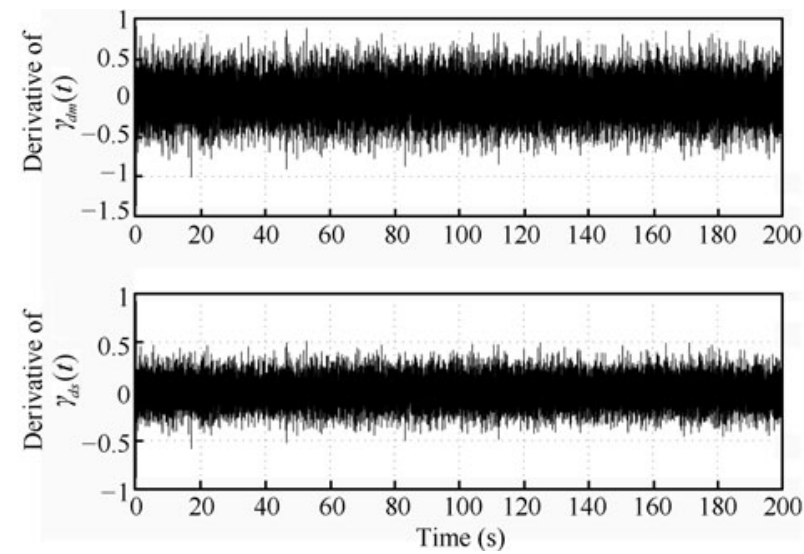

Fig. 7 The time derivative of unsymmetrical forward $\gamma_{d m}(t)$ and backward $\gamma_{d s}(t)$ random time varying communication delays in second


Fig. 8 Position tracking of the master and slave manipulator with random time varying delays with very hard interaction between remote slave and environment

\section{Conclusions}

In this paper, passive bilateral teleoperation systems have been studied with symmetrical and unsymmetrical time varying communication networks delay. A delayed position and position-velocity signals are used to couple local and remote sites via using telecommunication channel. Nonlinear adaptive control terms have been employed locally to learn and compensate uncertain parameters associated with gravity loading vector of the master and slave manipulators. Detailed stability analysis for three different control methods has been given by using Lyapunov-Krasovskii function to explore asymptotic convergence property of the closed loop teleoperators for both symmetric and unsymmetrical time varying delays. Simulation results have been used to demonstrate the theoretical development of this paper.

\section{Acknowledgements}

Authors thank anonymous reviewers for their constructive comments on our original submission.

\section{References}

[1] I. G. Polushin, P. X. Liu, C. H. Lung. A control scheme for stable force-reflecting teleoperation over IP networks. IEEE Transactions on Systems, Man, and Cybernetics, Part B: Cybernetics, vol. 36, no. 4, pp. 930-939, 2006.

[2] I. G. Polushin, P. X. Liu, C. H. Lung. A force-reflection algorithm for improved transparency in bilateral teleoperation with communication delay. IEEE/ASME Transactions on Mechatronics, vol. 12, no. 3, pp.361-374, 2007.

[3] C. C. Hua, P. X. Liu. Convergence analysis of teleoperation systems with unsymmetric time-varying delays. IEEE Transactions on Circuits and Systems II: Express Briefs, vol. 56, no. 3, pp. 240-244, 2009.

[4] C. C. Hua, P. X. Liu. Teleoperation over the internet with/without velocity signal. IEEE Transactions on Instrumentation and Measurement, vol. 60, no. 1, pp. 4-13, 2011.

[5] P. Arcara, C. Melchiorri. Control schemes for teleoperation with time delay: A comparative study. Robotics and $\mathrm{Au}-$ tonomous Systems, vol. 38, no. 1, pp. 49-64, 2002.

[6] P. F. Hokayem, M. W. Spong. Bilateral teleoperation: An historical survey. Automatica, vol. 42, no. 12, pp. 2035$2057,2006$.

[7] R. J. Anderson, M. W. Spong. Bilateral control of teleoperators with time delay. IEEE Transactions on Automatic Control, vol. 34, no. 5, pp. 494-501, 1989.

[8] R. J. Anderson, M. W. Spong. Asymptotic stability for force reflecting teleoperators with time delay. The International Journal of Robotics Research, vol. 11, no. 2, pp. 135-149, 1992.

[9] G. Niemeyer, J. J. E. Slotine. Telemanipulation with time delays. The International Journal of Robotics Research, vol. 23, no. 9, pp. 873-890, 2004.

[10] K. Kosuge, H. Murayama, K. Takeo. Bilateral feedback control of telemanipulators via computer network. In Proceedings of 1996 IEEE/RSJ International Conference on Intelligent Robots and Systems, IEEE, Osaka, pp. 1380-1385, 1996.

[11] J. H. Park, H. C. Cho. Sliding mode control of bilateral teleoperation systems with force-reflection on the Internet In Proceedings of 2000 IEEE/RSJ International Conference on Intelligent Robots and Systems, IEEE, Takamatsu, pp. 1187-1192, 2000.

[12] H. J. Li, A. G. Song. Virtual-environment modeling and correction for force-reflecting teleoperation with time delay. IEEE Transactions on Industrial Electronics, vol. 54 , no. 2 pp. 1227-1233, 2007.

[13] S. Munir, W. J. Book. Internet-based teleoperation using wave variables with prediction. IEEE/ASME Transactions on Mechatronics, vol. 7, no. 2, pp. 124-133, 2002.

[14] L. Bate, C. D. Cook, Z. Li. Reducing wave-based teleoperator reflections for unknown environments. IEEE Transactions on Industrial Electronics, vol. 58, no. 2, pp. 392-397, 2011.

[15] M. Alise, R. G. Roberts, D. W. Repperger, C. A. Moore, Jr., $\mathrm{S}$. Tosunoglu. On extending the wave variable method to multiple-DOF teleoperation systems. IEEE/ASME Transactions on Mechatronics, vol. 14, no. 1, pp. 55-63, 2009.

[16] N. Chopra, M. W. Spong, S. Hirche, M. Buss. Bilateral teleoperation over the internet: The time varying delay problem. In Proceedings of the American Control Conference, IEEE, Denver, Colorado, USA, pp. 155-160, 2003.

[17] Y. J. Pan, C. Canudas-de-Wit, O. Sename. A new predictive approach for bilateral teleoperation with applications to drive-by-wire systems. IEEE Transactions on Robotics, vol. 22, no. 6, pp. 1146-1162, 2006.

[18] D. J. Lee, M. W. Spong. Passive bilateral teleoperation with constant time delay. IEEE Transactions on Robotics, vol. 22, pp. 269-281, 2006.

[19] E. Nuno, R. Ortega, N. Barabanov, L. Basanez. A globally stable PD controller for bilateral teleoperators. IEEE Transactions on Robotics, vol. 24, no. 3, pp. 753-758, 2008

[20] D. Lee, K. Huang. Passive-set-position-modulation framework for interactive robotic systems. IEEE Transactions on Robotics, vol. 26, no. 2, pp. 354-369, 2010. 
[21] X. C. Shi, T. P. Zhang. Adaptive tracking control of uncertain MIMO nonlinear systems with time-varying delays and unmodeled dynamics. International Journal of Automation and Computing, vol. 10, no. 3, pp. 194-201, 2013.

[22] S. Islam, P. X. Liu, A. E. Saddik. Teleoperation over internet communication networks under time varying delay. In Proceedings of IEEE International Conference on $\mathrm{Au}$ tomation and Logistics, IEEE, Chongqing, China, pp. 1217, 2011.

[23] R. Kelly, V. Santibanez, A. Loria. Control of robot manipulators in joint space. Advanced Textbooks in Control and Signal Processing, New York: Springer-Verlag, 2005.

[24] N. Hogan. Impedance control: An approach to manipulation, Parts 1-3. Journal of Dynamic Systems, Measurement and Control, vol. 107, no. 1, pp. 1-24, 1985.

[25] D. E. Whitney. Historical perspective and state of the art in robot force control. In Proceedings of IEEE International Conference on Robotics and Automation, IEEE, St. Louis, MO, USA, vol. 2, pp. 262-268, 1985.

[26] H. K. Khalil. Nonlinear Systems, New York: Maxwell Macmillan, 1992.

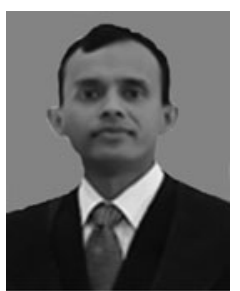

Shafiqul Islam earned his Ph. D. degree in electrical and computer engineering from Ottawa-Carleton Institute for electrical and computer engineering at the University of Ottawa and Carleton University, Canada. He has a master degree in control engineering and B.Sc. degree in electrical and electronic engineering. His research funded by many organizations including Natural Sciences and Engineering Research Council of Canada (NSERC), CMC Electronics, Carleton University, University of Ottawa, Lakehead University, Canada Research Chairs Program, etc. He was awarded Research Excellence in Science and Engineering from University of Ottawa, Canada, and Carleton University, Canada, for his outstanding contribution to research and development. He currently holds prestigious NSERC Canada visiting research fellowship award for visiting national and international research laboratory. He is the author of more than 50 peer reviewed journal and conference articles in the area of robotics and automation.

His research interests include distributed control of multiagent systems, consensus, formation, flocking/swarming and synchronization problems, cooperative robotics, nonlinear control theory and control of aerospace vehicles.

E-mail: sislam@sce.carleton.ca (Corresponding author)

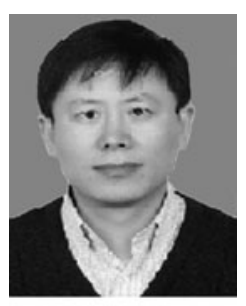

Xiaoping P. Liu received his Ph. D. degree from the University of Alberta in 2002. He has been with the Department of Systems and Computer Engineering, Carleton University, Canada since 2002 and is currently a professor and Canada Research Chair. He has published more than 200 research articles and serves as an associate editor for several journals including IEEE/ASME Transactions on Mechatronics and IEEE Transactions on Automation Science and Engineering. He received a 2007 Carleton Research Achievement Award, a 2006 Province of Ontario Early Researcher Award, a 2006 Carty Research Fellowship, the Best Conference Paper Award of the 2006 IEEE International Conference on Mechatronics and Automation, and a 2003 Province of Ontario Distinguished Researcher Award. He is a licensed member of the Professional Engineers of Ontario (P. Eng.) and a senior member of IEEE.
His research interests include interactive networked systems, haptics with application to medical simulations, robotics, control and intelligent systems, context-aware smart networks and wireless sensor networks.

E-mail: xpliu@sce.carleton.ca

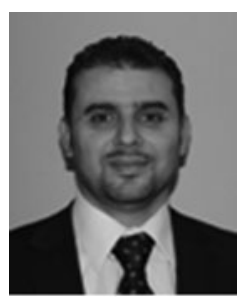

Abdulmotaleb El Saddik is a university research chair and professor in the School of Electrical Engineering and Computer Science at the University of Ottawa, is an internationally-recognized scholar who has made strong contributions to the knowledge and understanding of multimedia computing, communications and applications, particularly in the digitization, communication and security of the sense of touch, or haptics, which is a new medium that is significantly changing the way in which human-to-human and human-computer interactions are performed. He has authored and co-authored four books and more than 400 publications. He has received research grants and contracts totaling more than 18 millon. and has supervised more than 100 researchers. He received several international awards and is ACM Distinguished Scientist, fellow of the Engineering Institute of Canada, and fellow of the Canadian Academy of Engineers and fellow of IEEE.

His research interests include interactive media and serious games, biomedical engineering and biofeedback, haptic audio visual environments, social media, multimedia tele-surveillance, tangible user interface, collaborative ambient intelligence systems and applications.

E-mail: elsaddik@uottawa.ca

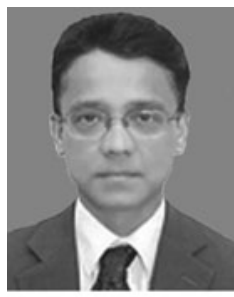

Lakmal Seneviratne earned his Ph. D. degree in mechanical engineering from King's College London (KCL). He has published over 250 peer reviewed articles on these topics. Before joining Khalifa University he was professor of mechatronics at King's College London. He was the founding director of the Centre for Robotics at KCL (1994-2010) and the head of Division of Engineering at KCL (2004-2009). He has been appointed as associate provost for Research and Graduate Studies of Khalifa University of Science, Technology and Research (KUSTAR), UAE.

His research interests include robotics and automation, with particular emphasis on increasing the autonomy of robotic systems interacting with complex dynamic environments.

E-mail: lakmal.seneviratne@kustar.ac.ae

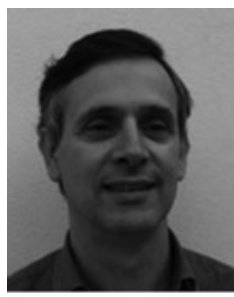

Jorge Dias has an "Agregacao" (Habilitation) degree and a $\mathrm{Ph} . \mathrm{D}$. degree on electrical engineering by the University of Coimbra, specialization in control and instrumentation. He has been an associated professor at the University of Coimbra with activities in the Department of Electrical Engineering and Computers (www.deec.uc.pt) and the Institute of Systems and Robotics (ISR) (www.isr.uc.pt ) from the University of Coimbra (UC) (www.uc.pt). He coordinated the Mobile Robotics Laboratory from Instituto of Systems and Robotics and the Laboratory of Systems and Automation (LAS) (http://las.ipn.pt) from the Instituto Pedro Nunes (IPN) (www.ipn.pt). Since July 2011, he has been acting as associate professor from Robotics and Electrical and Computer Engineering at Khalifa University of Science \& Technology, Abu Dhabi, UAE.

His research interests include computer vision and robotics. E-mail: jorge.dias@kustar.ac.ae 